\title{
CÓMO LEER Y GENERAR PUBLICACIONES CIENTÍFICAS. DISEÑOS DE ESTUDIO CLÍNICOS Y EPIDEMIOLÓGICOS: CONSIDERACIONES PARA LA INVESTIGACIÓN EN PEDIATRÍA CLINICAL AND EPIDEMIOLOGICAL STUDY DESIGNS: CONSIDERATIONS FOR RESEARCH IN PEDIATRICS
}

\author{
Klga. PhD Karla Yohannessen V. ${ }^{1-2}$, Dr. Carlos Ubilla P. ${ }^{3}$ \\ 1.- Profesor Asistente, Departamento de Pediatría y Cirugía Infantil, Facultad de Medicina, Universidad de Chile \\ 2.-Profesor Asistente, Programa de Salud Ambiental, Escuela de Salud Pública, Universidad de Chile. \\ 3.-Profesor Asociado, Departamento de Pediatría y Cirugía Infantil, Facultad de Medicina, Universidad de Chile.
}

\section{INTRODUCCIÓN}

Cuando un pediatra 0 un profesional clínico que trabaja con niños, se plantea una pregunta 0 un problema de investigación, el siguiente paso que debiera dar es la revisión exhaustiva de la literatura con el fin de conocer en forma detallada el problema a investigar y cuál es la evidencia disponible acerca de este. A partir de la revisión de la literatura, el investigador descubrirá los vacíos de conocimiento acerca de su problema y, de esta manera, aquella pregunta inicial será mantenida 0 replanteada con un mayor fundamento. La revisión de la literatura, además, ayudará al investigador a identificar aquellos aspectos metodológicos que han sido abordados por otros grupos de investigación, en especial aspectos específicos de la investigación con niños, que suelen presentar diferencias y desafíos en comparación con la investigación con adultos y deben ser considerados al plantearse los objetivos de la investigación y su metodología. Una vez planteados los objetivos, se define el diseño de la investigación, siendo este punto el que será abordado de manera más detallada en el presente artículo considerando un enfoque en la investigación con niños.

Para comenzar, los diseños o tipos de estudios pueden tener diferentes características según los cinco aspectos mostrados en la Tabla 1. Estos aspectos se entremezclan para entregar diferentes alternativas para responder a una pregunta de investigación, y al momento de planificar su estudio, el investigador deberá tener un conocimiento detallado del problema a investigar, lo que le permitirá elegir la alternativa más adecuada y enfocar su elección a la investigación en población pediátrica (1-6).

Por otro lado, antes de hacer una descripción de los diseños específicos, es importante conocer los diferentes propósitos que puede tener una investigación, lo cual irá ligado al tipo de diseño a utilizar. La Tabla 2 muestra los tipos de propósito en una investigación y su
RESUMEN

El diseño y la planificación de una investigación requiere conocer detalladamente el problema de estudio y las formas que se han abordado para investigarlo. Identificar el propósito del estudio ayudará a elegir el diseño más apropiado, determinar el o los grupos a estudiar, el conjunto suficiente de variables a evaluar y, en el caso de los pediatras o profesionales clínicos que trabajan con niños, tomar en cuenta las consideraciones especiales que se deben tener cuando se realiza investigación en pediatría. En este artículo se describen los tipos de diseño de estudio clínicos y epidemiológicos, con enfoque en la investigación en pediatría.

Palabras claves: Diseño de estudio, investigación, pediatría, metodología.

ABSTRACT

The design and planning of an investigation requires a detailed knowledge of the study problem and the ways that have been approached to investigate it. Identifying the purpose of the study will help choose the most appropriate design, determine the group (s) to study, the sufficient set of variables to evaluate and, in the case of pediatricians or clinical professionals who work with children, take into account special considerations. to have when conducting pediatric research. This article describes the types of clinical and epidemiological study design with a focus on pediatric research.

Keywords: Study design, research, pediatrics, methodology

Tabla 1. Principales características de los tipos de diseños de estudios.

\begin{tabular}{lll} 
Caracteristica & Descripción & Tipos \\
\hline Hipótesis & $\begin{array}{l}\text { Presencia o ausencia } \\
\text { de hipótesis }\end{array}$ & $\begin{array}{l}\text { Descriptivo: describen una situación, generan hipótesis } \\
\text { Analítico: evalúan una presunta relación causal, comprueban } \\
\text { hipótesis }\end{array}$ \\
\hline $\begin{array}{lll}\text { Asignación de } \\
\text { la exposición }\end{array}$ & $\begin{array}{l}\text { Forma en que los suje- } \\
\text { tos son expuestos a la } \\
\text { variable independiente }\end{array}$ & $\begin{array}{l}\text { Experimental: la exposición es asignada a los participantes } \\
\text { de forma aleatoria } \\
\text { Observacional: el investigador no interviene en la exposi- } \\
\text { ción, solo la observa (mide). }\end{array}$ \\
\hline$N^{\circ}$ mediciones & $\begin{array}{l}\text { Cantidad de medicio- } \\
\text { nes realizadas a cada } \\
\text { unidad de estudio }\end{array}$ & $\begin{array}{l}\text { Longitudinal: las variables se miden varias veces en el } \\
\text { tiempo. } \\
\text { Transversal: las variables se miden una sola vez, sin } \\
\text { seguimiento. }\end{array}$ \\
\hline Temporalidad & $\begin{array}{l}\text { Secuencia temporal } \\
\text { con respecto al proble- } \\
\text { ma estudiado }\end{array}$ & $\begin{array}{l}\text { Prospectivo: las variables estudiadas se miden o registrana } \\
\text { medida que suceden } \\
\text { Retrospectivo: las variables estudiadas ya fueron evaluadas } \\
\text { o registradas, por lo tanto, los datos se obtienen a partir de } \\
\text { archivos o registros. }\end{array}$ \\
\hline $\begin{array}{lll}\text { Unidad } \\
\text { análisis }\end{array}$ & $\begin{array}{l}\text { A partir de qué } 0 \\
\text { quiénes se obtendrán } \\
\text { los datos }\end{array}$ & $\begin{array}{l}\text { Individual: las unidades de observación son los individuos } \\
\text { Grupal: la unidad de observación son unidades geográficas } \\
\text { u hospitalarias. }\end{array}$
\end{tabular}

\section{Autor para correspondencia:}

Karla Yohannessen V., Departamento de Pediatría y Cirugía Infantil, Facultad de Medicina,

Universidad de Chile. Independencia 1027, Independencia, Santiago, Chile. Teléfono: +562 29786539

Correo electrónico: karlayohannessen@med.uchile.cl 
descripción. Martinez-Castaldi et al. (7) estudiaron la frecuencia de los tipos de propósitos en la investigación clínica publicada comparando entre niños y adultos, concluyendo que los estudios en los que participaron adultos tenían una probabilidad significativamente mayor de ser estudios de terapia, mientras que los niños tenían una probabilidad significativamente mayor de participar en estudios epidemiológicos.

A continuación, se detallarán los tipos de diseño de estudio clínicos y epidemiológicos, diferenciándolos según la ausencia (estudios descriptivos) y la presencia (estudios analíticos) de hipótesis, haciendo énfasis en los estudios analíticos y los desafíos que plantean estos cuando se realiza investigación en pediatría.

\section{DISEÑOS DE ESTUDIO DESCRIPTIVOS}

El principal objetivo de estos estudios es describir la frecuencia y las características de un problema de salud en una población, responden a las preguntas ¿Quiénes?, ¿Dónde?, ¿Cuándo? y ¿Cómo? También pueden ser utilizados para describir una relación entre dos o más variables, sin embargo, no se podrá asumir una relación causal entre ellas. En ambos casos, estos estudios sirven para generar hipótesis sensatas, que en una etapa posterior podrán ser comprobadas a través de estudios analíticos (2-6). Entre los estudios descriptivos destacan los reportes de casos individuales, las series de casos y los estudios transversales descriptivos.

Los reportes de casos son un tipo de diseño bastante utilizado en estudios clínicos. Estos son relatos que describen la ocurrencia de un problema de salud destacado o poco frecuente (6), el cual puede tener relación con casos de enfermedades raras o con enfermedades comunes que presentan evoluciones inesperadas y cuya información puede ser útil desde el punto de vista epidemiológico o clínico para orientar el actuar de otros profesionales frente a situaciones similares.

De manera similar, las series de casos, son un conjunto más grande de casos de enfermedad, a menudo agrupados en forma consecutiva y ordenado según características comunes, que orientarán a los profesionales en la conducta a seguir con pacientes que presenten el mismo problema de salud (6). Estos estudios describen la experiencia de un grupo de sujetos con un diagnóstico o evolución similar, generalmente de baja frecuencia. Usualmente, se describen las características de los propios sujetos y además los aspectos de la enfermedad, tratamiento y/o evolución, información que sirve para generar nuevas hipótesis. Ambos diseños son estudios de tipo observacional

Tabla 2. Diferentes tipos de propósitos del estudio y descripción

\begin{tabular}{ll} 
Propósito del estudio & Descripción \\
\hline Terapia & $\begin{array}{l}\text { Determinar el efecto de diferentes tratamientos para mejorar la función del } \\
\text { paciente o evitar eventos adversos. }\end{array}$ \\
\hline Diagnóstico & $\begin{array}{l}\text { Establecer el comportamiento de una intervención para diferenciar entre } \\
\text { personas con y sin la enfermedad. }\end{array}$ \\
\hline $\begin{array}{l}\text { Descripción de manifesta- } \\
\text { ciones de la enfermedad }\end{array}$ & $\begin{array}{l}\text { Describir y/o comparar signos y síntomas de condiciones de salud o enfer- } \\
\text { medades (diagnóstico diferencial). }\end{array}$ \\
\hline Pronóstico & $\begin{array}{l}\text { Estimar el curso futuro de la enfermedad e identificar factores que modifi- } \\
\text { can el pronóstico. }\end{array}$ \\
\hline Daño & $\begin{array}{l}\text { Determinar efectos de agentes potencialmente dañinos (incluidas las } \\
\text { terapias) sobre la función del paciente. }\end{array}$ \\
\hline Epidemiológico & $\begin{array}{l}\text { Cuantificar la prevalencia o incidencia de una enfermedad o factor de ries- } \\
\text { go. Estimar asociaciones entre factores de riesgo y enfermedades. }\end{array}$ \\
\hline Evaluación de servicios & $\begin{array}{l}\text { Cuantificar el costo y la calidad en la prestación de atención médica. } \\
\text { de salud } \\
\text { Estudiar proveedores, sistemas de atención médica y los propios servicios } \\
\text { de salud. }\end{array}$
\end{tabular}

cuya unidad de análisis es individual, pueden ser prospectivos o retrospectivos y, en general, son longitudinales dado que muestran en detalle la evolución de algunos individuos o de un grupo de individuos. Estos diseños se utilizan con el propósito de realizar la descripción de manifestaciones de la enfermedad, y el principal desafío de estos diseños, aplicados en pediatría, será obtener tanto la aprobación del comité de ética y el consentimiento informado de los padres o tutores, así como el asentimiento de los niños a partir de cierta edad, en especial si el estudio considera hacer procedimientos evaluativos extras, fuera de los que se realizan rutinariamente para la enfermedad específica, o si se utilizarán fotografías o imágenes de los sujetos de estudio.

Los estudios transversales descriptivos se utilizan para describir la frecuencia (y estimar la prevalencia: proporción de la población que presenta el evento en estudio en un momento determinado en el tiempo) y distribución de eventos de salud y enfermedad $(6,8)$. Son estudios observacionales y su unidad de análisis es individual. Estos hacen una evaluación de una sola vez de la presencia de la enfermedad o los factores de riesgo en un grupo de estudio que, idealmente, ha sido elegido al azar de la población de interés (6). La información generada a partir de estos estudios es de gran utilidad para valorar el estado de salud de una población, comunidad o grupo de pacientes y determinar sus necesidades $(5,6,9)$, por lo que este diseño puede cumplir con los propósitos de descripción de manifestaciones de la enfermedad, epidemiológico y de evaluación de servicios de salud.

\section{DISEÑOS DE ESTUDIO ANALÍTICOS}

Los diseños de estudio analíticos evalúan hipótesis relacionadas con asociaciones entre posibles exposiciones a ciertas intervenciones o factores de riesgo y las enfermedades o problemas de salud $(2,3)$, generalmente intentan responder la pregunta: ¿Por qué? La evaluación de la evidencia generada a partir de los estudios analíticos se centrará en aspectos del diseño utilizado, la forma de asignación de la exposición y selección de sujetos, la ejecución y el análisis, aspectos que pueden introducir sesgos que distorsionan la verdadera relación que exista entre la exposición y la enfermedad.

En este tipo de estudios la forma de selección de los participantes que conformarán la muestra o los grupos de estudios debiera garantizar la comparación de estos, en especial en aquellas variables que puedan tener alguna relación con la exposición o enfermedad de interés, y una forma de lograrlo es realizando una asignación (de la exposición) o selección (de los sujetos) aleatoria.

Los estudios analíticos se utilizan principalmente para responder a propósitos de terapia, diagnóstico, pronóstico, daño y epidemiológicos. No obstante, en población pediátrica se deben tener en cuenta varios aspectos y consideraciones especiales, algunos de estos se detallan en la Tabla 3. A continuación, se describen los estudios analíticos más utilizados.

\section{Estudio experimental}

En estos estudios el investigador es quien asigna y controla la exposición (experimento); son prospectivos e implican un seguimiento de los participantes de acuerdo a un protocolo determinado $(4,5)$. Los aspectos éticos en estos estudios son fundamentales debido a que se realiza una intervención en un grupo de individuos, por ejemplo: tratamientos farmacológicos, actividades preventivas, suspensión de algún tratamiento, entre otros, y no 
debieran utilizarse para estudiar intervenciones que sean potencialmente perjudiciales (4).

La asignación de la exposición o intervención se debe realizar en forma aleatoria. El objetivo de esto es asegurar que ambos grupos (intervenido y no intervenido) sean comparables, al distribuir equilibradamente las variables tanto conocidas como desconocidas, y evitar que las preferencias, tanto de los individuos como de los encargados del estudio, interven- gan en la asignación de la exposición, disminuyendo los posibles sesgos de selección (por ejemplo, la intención de tratar a pacientes que tienen ciertas características) $(4,5)$. Por otro lado, las expectativas de los pacientes y los investigadores pueden influir en la evaluación de la respuesta observada, lo cual se evita utilizando técnicas de enmascaramiento (o ciego), así los sujetos que están relacionados con el estudio desconocen los hechos u observaciones

Tabla 3. Aspectos y consideraciones en la planificación de los estudios pediátricos.

\begin{tabular}{l} 
Aspecto \\
\hline Subpoblaciones pediátri- \\
cas: considerar el grupo \\
de edad que participará \\
del estudio (recién na- \\
cidos, recién nacidos \\
prematuros, lactantes y \\
niños pequeños, niños, \\
adolescentes). \\
\hline Diseño del estudio: \\
usar protocolos para la \\
población pediátrica y no \\
reelaborarse a partir de \\
protocolos para adultos.
\end{tabular}

\section{Consideraciones}

Considerar la edad, el desarrollo físico, la enfermedad, la dosis, la frecuencia de dosificación, la duración del tratamiento y la vía de administración. Si necesita formulaciones pediátricas:

- Fáciles de administrar y tragar.

- Aceptables en sabor y volumen

- Dosis adecuadas y concentración.

- Biodisponibilidad adecuada.

- Administración poco frecuente.

Personalizar el diseño para las poblaciones específicas

- Definición adecuada del desenlace pediátrico relevante (evitar múltiples desenlaces sin fundamento).

- Identificar correctamente las dosis para estudios de eficacia

- Identificar problemas de factibilidad.

- Muestra pequeña o bajo reclutamiento, la variabilidad del desarrollo debe tenerse en cuenta en los criterios de inclusión, los cálculos del tamaño muestral y análisis.

- El diseño debe ser realista para que las familias se comprometan, los diseños inadecuados llevarán a una inscripción lenta y baja retención (mayor costo y retrasos en la aprobación del protocolo de estudio).

Uso del placebo: suele ser . Si bien no es ideal, el uso de un placebo puede ser aceptable si no hay más restringido porque los niños no pueden dar su consentimiento. terapias aprobadas o estudiadas adecuadamente.

- Minimizar la exposición al placebo: n de sujetos, duración del estudio, necesidad predefinida de criterios de interrupción.

\begin{tabular}{|c|c|}
\hline $\begin{array}{l}\text { Criterios de valoración } \\
\text { específicos pediátricos: } \\
\text { evitar múltiples desenla- } \\
\text { ces y mediciones. }\end{array}$ & $\begin{array}{l}\text { - Medidas fisiológicas y biomarcadores (peso, estatura, hormona tiroidea, } \\
\text { crecimiento esquelético, maduración sexual, etc.). } \\
\text { - Desarrollo y función cognitiva (efectos a corto y largo plazo en atención, } \\
\text { memoria y aprendizaje, habilidad lingüística, etc.). } \\
\text { - Maduración conductual y psicológica (considerar los hitos: lista de verifica- } \\
\text { ción del comportamiento infantil calidad de vida rendimiento escolar etc) }\end{array}$ \\
\hline
\end{tabular}

Duración del tratamiento, seguimiento y retención - Minimizar la intensidad y frecuencia de las evaluaciones del estudio

- Limitar el número y volumen de extracciones de sangre: hacer coincidir la recolección de muestras de sangre especificadas en el protocolo con muestras de rutina.

- Limitar el procedimiento invasivo tanto como sea posible.

- Minimizar el dolor y la angustia: uso anestesia tópica, agujas de mariposa, tubos de muestreo pediátricos.

- Tener en cuenta no solo al niño, sino también a los padres y hermanos, al planificar la frecuencia y duración de las evaluaciones.

Consideraciones éticas y protecciones especiales en la investigación pediátrica.
- Las protecciones especiales en las poblaciones vulnerables es una prioridad para los comités de ética, que debe considerar.

- La evaluación del comité de ética revisará la categoría de riesgo de la investigación: proceso de consentimiento - permiso de los padres, monitoreo de la seguridad de los datos, continuidad del tratamiento después del estudio clínico, limitación del uso de placebos.

- Los padres aceptan los riesgos e inconvenientes potenciales, pero éstos pueden afectar al niño

- Planificar de antemano si se solicitará el asentimiento pediátrico y como se realizará

- Formularios: considerar tener uno o más formularios de asentimiento separados del formulario de consentimiento de los padres, deben estar dirigidos al nivel de grado de lectura apropiado para la persona más joven que firmará. que tienen relación con la intervención recibida, evitándose de esta forma la introducción de sesgos en los resultados.

Un aspecto importante a tener en cuenta en estos estudios prospectivos, es que son susceptibles a sesgos por pérdidas de sujetos durante el seguimiento. Además, cuando las condiciones en las cuales se realiza la intervención son muy restringidas, la aplicación de sus conclusiones en condiciones reales puede presentar una respuesta distinta, por ende, podrían tener baja validez externa (generalización de los resultados a toda la población) $(4,5)$.

El diseño experimental más conocido es el ensayo clínico aleatorio, en el cual se conforman dos grupos de pacientes, uno que recibe la intervención y otro que actúa como control con fines de tener una referencia para hacer comparaciones (Figura 1) (4). Otro tipo de estudio experimental son las pruebas de campo, en las cuales se utilizan sujetos "sanos" (sin la enfermedad), usualmente para evaluar intervenciones preventivas, como las vacunas (4).

\section{Estudio de cohorte}

Estos estudios son de tipo observacional (sin experimento) y longitudinal (al menos dos mediciones), en los que se comparan dos cohortes cuyos integrantes difieren por su exposición al factor de estudio (exposición) con el objetivo de evaluar una posible relación causa-efecto $(4,9,10)$. Son útiles para estudiar exposiciones poco frecuentes y/ o evaluar múltiples resultados, mientras que son de menor utilidad en enfermedades poco frecuentes.

Al comienzo del estudio, los sujetos se clasifican según el(los) factor(es) de exposición de interés en grupos expuesto y no expuesto, y ambos grupos se siguen en el tiempo para comparar la ocurrencia del evento/enfermedad de interés en los grupos. La validez de estos estudios depende en gran parte del supuesto de que ambos grupos son comparables respecto a otros factores asociados a la exposición o el evento de interés. Todos los sujetos deben estar en riesgo de presentar el evento de interés, es decir, deben estar libres del evento / enfermedad al inicio del estudio. Por esta razón, se postula que la causa precede a la ocurrencia del evento (ej: enfermedad) y que la enfermedad o cualquier factor asociado a esta no influyó en forma diferencial en la selección o determinación de la exposición (10).

Una gran fortaleza de los estudios de cohortes es que permiten obtener una información detallada, precisa y objetiva de la exposición en estudio. Esta medición detallada hace posible estudiar el efecto en un subgrupo de sujetos que pueden estar expuestos en diferentes grados, y de esta manera evaluar una 
posible relación exposición-respuesta (4).

En los estudios de cohorte, al igual que en los ensayos clínicos, es posible estimar el Riesgo Relativo (RR), debido a que se puede obtener la incidencia acumulada o la tasa de incidencia en los grupos expuesto y no expuesto (casos nuevos dividido por el total de la población en riesgo o por el tiempo libre del evento) (Figura 2). Su interpretación general dependerá de si su valor es mayor o menor que el 1 (valor sin asociación), si el RR es mayor que 1 indica que el factor en estudio se comporta como factor de riesgo y si es menor que 1 que el factor en estudio se comporta como protector.

La principal desventaja de estos estudios es la pérdida de participantes durante el seguimiento lo que puede influir en la precisión y validez de sus resultados, en especial si las pérdidas son más marcadas en uno de los grupos estudiados. Por otro lado, pueden existir cambios en la exposición durante el seguimiento, lo cual debe ser registrado en forma detallada. $(4,9,10)$

\section{Estudio de caso-control}

Se caracterizan porque su criterio de selección de grupos de estudio se basa en la presencia de un efecto o enfermedad determinada (casos) y su ausencia (controles) $(4,5,11)$. Luego, ambos grupos se comparan con respecto a la frecuencia de la exposición (en el pasado) a uno o más factores de riesgo que se están estudiando, en que el investigador sospecha que tienen relación con la presencia de la enfermedad.

Son una alternativa costo-efectiva para identificar factores de riesgo y generar hipótesis para estudios posteriores. No obstante, como los sujetos ya presentan el efecto (o enfermedad) al inicio del estudio, no se puede estimar directamente la incidencia o la prevalencia, por lo que son limitados para establecer causalidad. Por otro lado, la búsqueda de la posible causa en el pasado puede introducir errores en su medición y sesgar los resultados $(2,3,5,9)$.

En este diseño, los investigadores deberán ser rigurosos en realizar una correcta clasificación de los individuos con respecto a la condición de enfermedad, por lo que se deberá establecer de manera clara y explícita la definición de la enfermedad y los criterios que deben cumplir los sujetos que serán clasificados como enfermos (casos) y no enfermos (controles). Una vez establecidos los criterios para identificar los casos, estos deben tener la misma probabilidad de ser incluidos en el estudio. Para la selección de los casos se podrán utilizar poblaciones de casos incidentes (nuevos), casos prevalentes (existentes) o casos fallecidos. Por

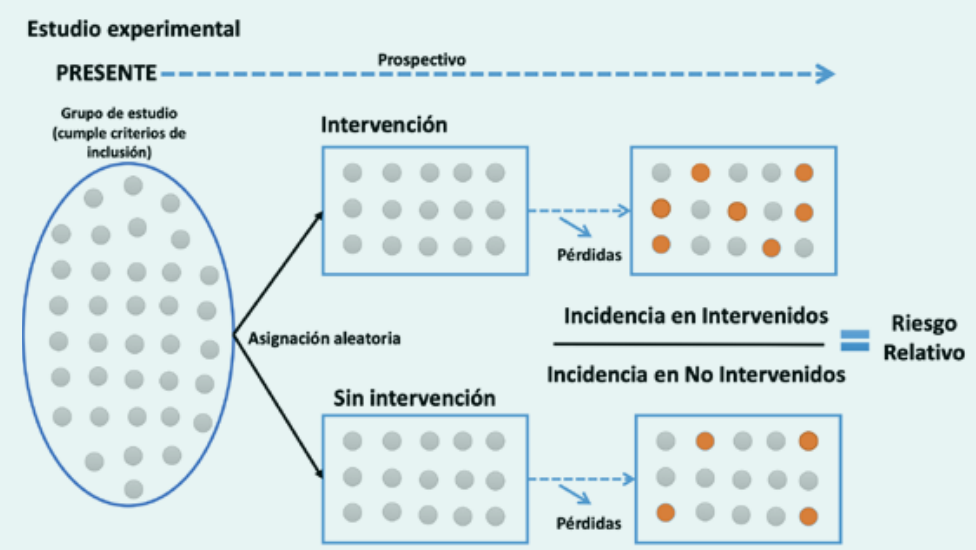

Figura 1. Esquema clásico de un ensayo clínico aleatorizado. Se conforman 2 grupos de pacientes sin el evento o enfermedad (círculos grises), ambos grupos son seguidos a lo largo del tiempo observando la aparición del evento o las respuestas frente a la intervención (círculos anaranjados), obteniendo la incidencia en cada grupo y el riesgo relativo como medida de asociación.

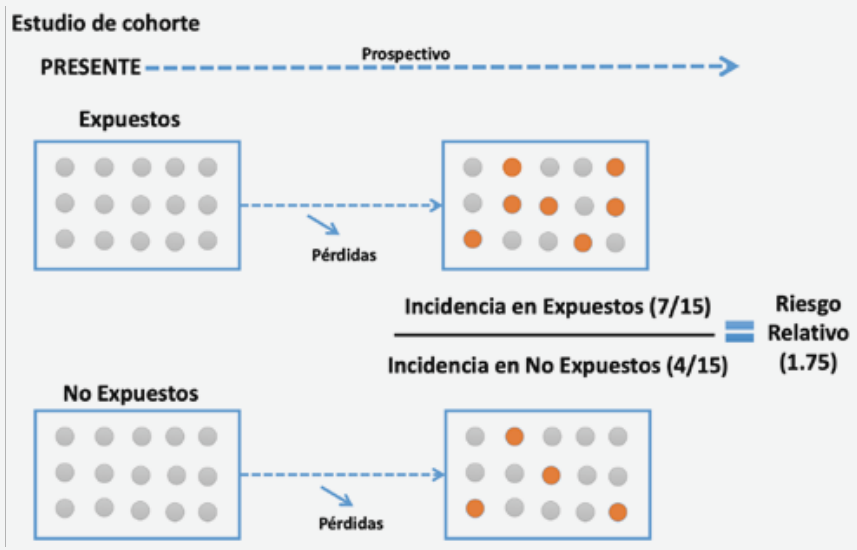

Figura 2. Esquema clásico de un estudio de cohorte. En este esquema se siguió durante un determinado tiempo a 15 sujetos expuestos y 15 no expuestos al factor de riesgo, el cual no presento pérdidas de sujetos durante su desarrollo (cohorte ideal). La incidencia en el grupo expuesto se calculó dividiendo los casos (círculos anaranjados) que aparecieron en el grupo expuesto (numerador=7) sobre el total de sujetos expuestos (denominador=15), mientras que la incidencia en el grupo no expuesto se calculó dividiendo los casos (numerador=4) sobre el total de sujetos no expuestos (denominador=15). La incidencia en los expuestos al factor de riesgo es mayor que la incidencia los no expuestos. El RR se calculó dividiendo la incidencia en el grupo expuesto (numerador=7/15) sobre la incidencia en el grupo no expuesto (denominador=4/15), resultando un $R R=1.75$, lo que significa que la exposición está asociada en forma positiva con el evento/enfermedad o que los expuestos tienen $75 \%$ más probabilidad de presentar el evento/ enfermedad que el grupo no expuesto.

otra parte, la selección del grupo control es clave en este tipo de estudios debido a que debe ser comparable al grupo de los casos. Es crucial que los controles sean representativos de la población de donde provienen los casos (2-4). Una vez definidas las poblaciones tanto de casos como controles, lo óptimo es que la selección sea a través de un muestreo aleatorio.

Por otro lado, la secuencia temporal de los estudios de casos y controles obliga a la obtención de información de la exposición en el pasado (retrospectivo) y, por lo tanto, la forma de medirla deberá ser cuidadosa y lo más objetiva posible. La selección de la fuente de información dependerá de lo que se desea medir, lo más frecuente es la utilización de cuestionarios y encuestas auto-aplicadas o aplicadas por entrevistadores. También, se pueden utilizar 

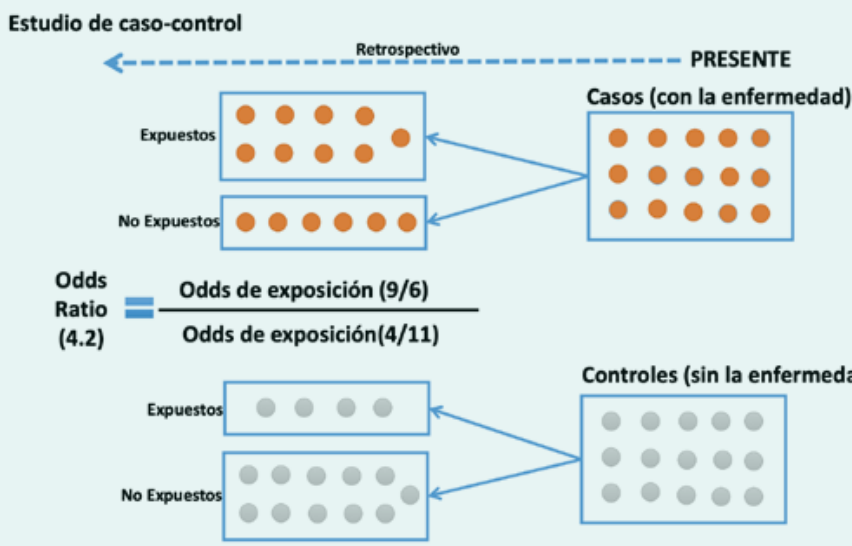

Figura 3. Esquema clásico de un estudio de casos y controles. En este esquema se seleccionaron 15 sujetos con la enfermedad de interés (casos, círculos anaranjados) y 15 sin la enfermedad de interés (controles, círculos grises), a quienes se les evaluó la presencia del factor de riesgo en el pasado. El odds de exposición en los casos se calculó dividiendo los casos que estuvieron expuestos (numerador=9) sobre los casos que no estuvieron expuestos (denominador=6). Luego, el odds de exposición en los controles se calculó dividiendo los controles que estuvieron expuestos (numerador=4) sobre los controles que no estuvieron expuestos (denominador=11). El OR se calculó dividiendo el odds de exposición de los casos (numerador=9/6)) sobre el odds de exposición de los controles (denominador=4/11), lo que resulta en $\mathrm{OR}=4.2$. Esto significa que los casos tienen 4.2 veces más posibilidades de haber estado expuestos al factor de riesgo que los controles.

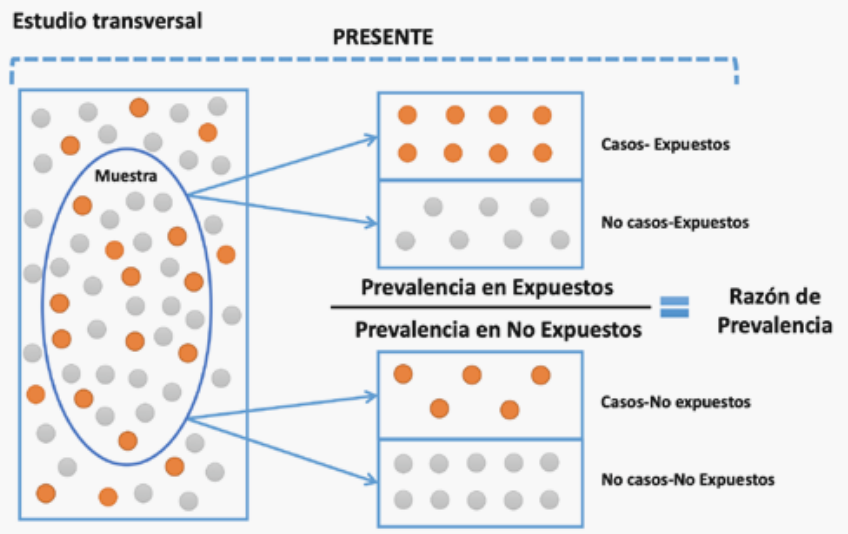

Figura 4. Esquema clásico de un estudio transversal. En la muestra se mide la exposición y la enfermedad al mismo tiempo. La RP se calcula dividiendo la prevalencia de la enfermedad en los expuestos sobre la prevalencia de la enfermedad en los no expuestos.

medidas biológicas si éstas son estables en el tiempo y en algunas ocasiones se puede obtener la información de personas próximas a los sujetos de estudio (familiares, cuidadores, etc.) (4).

A diferencia de los estudios de cohorte, en los estudios de casos y controles no se puede estimar directamente la incidencia de la enfermedad en expuestos y no expuestos; en este caso el estimador para medir la asociación entre la exposición y la enfermedad es el Odds
Ratio (OR). Para estimar el OR, primero se calculan los odds de exposición en los casos y en los controles, es decir, se compara la posibilidad de ocurrencia de un evento con la posibilidad de que no ocurra bajo las mismas condiciones (Figura 3). La interpretación general del OR dependerá de si su valor es mayor o menor que 1 , si el OR es mayor que 1 indica que el factor en estudio se comporta como factor de riesgo y si es menor que 1 que el factor en estudio se comporta como protector.
Las principales ventajas de estos estudios es que sirven para estudiar enfermedades 0 eventos de salud poco frecuentes, y se pueden evaluar de forma simultánea varios posibles factores de riesgo o de protección. No obstante, son bastante susceptibles a sesgos que están relacionados con la selección de los casos y los controles, y sesgos de información debido a que la exposición se indaga en el pasado (11).

\section{Estudio transversal analítico}

Es un tipo de estudio observacional cuya unidad de análisis es el individuo. En estos se realiza una evaluación simultánea y de una sola vez, de la presencia (y ausencia) de la enfermedad y la exposición en un grupo de estudio (Figura 4), lo cual no permite conocer la secuencia temporal de los acontecimientos y, por lo tanto, no es posible determinar si la exposición precedió a la enfermedad o viceversa $(4,6,8)$. Esta limitación para establecer causalidad es compensada por su flexibilidad para explorar asociaciones entre múltiples exposiciones y múltiples efectos. Son estudios muy comunes y útiles, puesto que entregan información importante para la planificación y administración de recursos económicos en salud (8).

La medida de asociación que se puede obtener a partir de un estudio transversal analítico es la Razón de Prevalencias (RP), su interpretación es similar al RR y OR, sin embargo se debe tener presente de que ambas mediciones (enfermedad y exposición) fueron realizadas al mismo tiempo, y por esta razón no se tendrá la certeza de que la exposición precedió a la enfermedad. Otra medida que se puede obtener es el OR, su valor se aproximará al de la RP si la prevalencia de la enfermedad es baja.

\section{ESTUDIOS ECOLÓGICOS}

En los estudios ecológicos, que pueden ser descriptivos o analíticos, se compara la frecuencia de la enfermedad o exposición entre unidades geográficas u hospitalarias en un mismo período, o su variación en el tiempo en una misma unidad geográfica u hospitalaria, cuyo propósito es buscar patrones espaciales o temporales que sugieran hipótesis de las causas (12). En estos estudios la unidad de observación y análisis es grupal, lo cual estará definido según la unidad(es) geográfica(s). La distribución de las características estudiadas a nivel individual es desconocida, por lo tanto, las conclusiones de estos estudios solo podrán referirse a la(s) unidad(es) estudiada(s). Esto se conoce como falacia ecológica, un sesgo inherente a este tipo de estudio $(3,12)$.

Una ventaja de los estudios ecológicos 
es que las variables que se utilizan son de tipo "agregada" o grupales, que son medidas de resumen que representan a la unidad estudiada, por ejemplo, la prevalencia de asma en cada comuna o el promedio de días de ausentismo escolar por una condición especifica según colegios. Usualmente existe algún registro de los datos o están disponibles en los sitios web de ministerios de salud, institutos de estadísticas y las encuestas poblacionales, entre otras $(2$, 3), por lo que son rápidos y de bajo costo $(4,12)$.

\section{CONCLUSIONES}

Finalmente, la selección de la mejor alternativa entre los diseños de estudios dependerá no solo de la pregunta de investigación, sino que también de la frecuencia de la enfermedad o de exposición en la población, del tiempo disponible para realizar el estudio, así como los recursos económicos que se destinen para llevar a cabo el estudio. Además, en la planificación del estudio se deberán considerar aquellos aspectos relevantes de la investigación en pediatría
REFERENCIAS

1. Hernández-Ávila M, Garrido-Latorre F, López-Moreno S Diseño de estudios epidemiológicos. Salud Publica de México 2000; 42(2): 144-154. ISSN: 0253-1751

2. Szklo M, Nieto F.J. Epidemiología intermedia: conceptos y aplicaciones. Díaz de Santos, Madrid, España, 2003.

3. Szklo M\& Nieto F.J. Epidemiology: beyond the basics. Jones \& Bartlett Learning, Estados Unidos, 2019.

4. Argimón-Pallás J, Jiménez-Villa J. Métodos de investigación clínica y epidemiológica. Elsevier España, España, 2013.

5. Gordis L. Epidemiología. Elsevier España, España, 2005

6. Friis R. Epidemiology 101. Jones \& Bartlett Learning, Estados Unidos, 2010

7. Martínez-Castaldi C, Silverstein M, Bauchner H. Child Versus Adult Research: The Gap in High-Quality Study Design Pediatrics 2008; 122(1):52-7. DOI: 10.1542/peds.20072849.

8. Hernández B, Velasco H. Encuestas transversales. Salud Pública de México 2000 : 42(5): 447-455. ISSN:0253-1751

9. Savitz, D. Interpreting Epidemiologic Evidence Strategies for Study Design and Analysis. Oxford University Press, Estados Unidos, 2003

10. Lazcano-Ponce E, Fernández E, Salazar-Martinez E, Hernández-Ávila M. Estudios de cohorte. Metodología, sesgos y aplicación. Salud Publica de México 2000; 42(3): 230-241 ISSN: 0036-3634

11. Lazcano-Ponce E, Salazar-Martinez E, Hernández-Ávila M. Estudios epidemiológicos de casos y controles. Fundamento Teórico, variantes y aplicaciones. Salud Publica de México 2001: 43(2): 135-150. ISSN: 0036-3634

12. Borja-Aburto V. Estudios ecológicos. Salud Pública de México 2000; 42(6): 533-538. ISSN: 0253-1751 